\title{
Algoritmo teórico para la estimación de la radiación solar global para una atmósfera despejada
}

\author{
Carlos Girado-Polo*, Leonardo Gónima-Gónima \\ Grupo de Física Aplicada y de Materiales, Departamento de Física y Electrónica, Universidad de Córdoba, Montería Colombia
}

\begin{abstract}
Resumen
En este trabajo se desarrolló un algoritmo para la estimación de la radiación solar global, para una atmósfera despejada, mediante el modelamiento de las ecuaciones teóricas de transferencia radiativa de onda corta $(0,3 \mu \mathrm{m}$ - 2,8 $\mu \mathrm{m})$. Se determinaron las transmitancias espectrales de la radiación solar directa, debidas a la dispersión de Rayleigh y Mie. Mediante el software Propiedades Ópticas de Aerosoles y Nubes (Optical Properties of Aerosols and Clouds, OPAC) se calculó la Profundidad Óptica de los Aerosoles (Aerosol Optical Depth, AOD) para cuatro diferentes tipos de atmósferas, indispensable para la determinación del índice de turbidez de Ångström. Así mismo, se calcularon la transmitancias espectrales por absorción de la radiación solar directa, incluyendo aerosoles, vapor de agua, ozono y aire seco (mezcla de gases). El contenido de $\mathrm{O}_{3}$ se obtuvo de los datos diarios existentes en base de datos de la NASA. Para la componente difusa de la radiación solar, se dedujo una nueva expresión para el cálculo de la fracción de la radiación solar dispersada por los aerosoles hacia la superficie terrestre. La comparación estadística entre los resultados obtenidos con el algoritmo desarrollado, los datos medidos de la radiación global (estación Potsdam - Alemania) y los resultados de otros tres modelos radiativos, entre 2012 y 2014, muestra que el nuevo modelo permite calcular los valores horarios de la radiación solar global con suficiente precisión. (C) 2018. Acad. Colomb. Cienc. Ex. Fis. Nat.

Palabras clave: Modelación teórica; Radiación solar global; Profundidad Óptica de los Aerosoles; Fracción espectral de dispersión hacia adelante; Radiación solar directa; Radiación solar difusa.
\end{abstract}

Theoretical algorithm for estimating global solar radiation for a cloudless atmosphere

\begin{abstract}
In this work we developed an algorithm to estimate global solar radiation for a cloudless atmosphere, by using the shortwave radiative transfer equations $(0.3 \mu \mathrm{m}-2.8 \mu \mathrm{m})$. Spectral transmittances of direct solar radiation due to Rayleigh and Mie scattering were determined. In order to estimate the Ångström turbidity coefficient, the Aerosol Optical Depth (AOD) was calculated for four different types of atmospheres, using the software Optical Properties of Aerosols and Clouds (OPAC). Likewise, spectral transmittances due to absorption of direct solar radiation were calculated, including aerosols, water vapor, carbon dioxide and dry air (mixed gases). The ozone content was obtained from daily data of NASA's database. For the diffuse component of solar radiation, a new equation was developed for the calculation of the spectral forward scattering fraction. The statistical comparison between the results obtained with the model developed here, the measured global solar radiation data at Potsdam radiation station in Germany and the results of other three radiative models, between 2012 and 2014, shows that the new model allows to calculate the hourly global solar radiation with sufficient precision. (C) 2018. Acad. Colomb. Cienc. Ex. Fis. Nat.
\end{abstract}

Key words: Theoretical modelling; Global solar radiation; Aerosol Optical Depth; Spectral forward scattering fraction; Direct solar radiation; Diffuse solar radiation.

\section{Introducción}

El primer algoritmo publicado para el cálculo de la radiación solar global fue el de Ångström (1924), que posteriormente fue modificado por Prescott (1940). En la actualidad, los modelos denominados Ångström-Prescott, que de acuerdo con Pelkowski (2009) incluyen de forma integral las interacciones entre la atmósfera despejada y la radiación solar, son utilizados debido a su sencillez y facilidad en el cálculo de la radiación solar. Estos modelos para poderlos utilizar, requieren indispensablemente de datos medidos "in situ" de la radiación solar global (i.e. López, et al., 2007, Janjai, et al., 2013, Pacheco, et al., 2017), sin la

*Correspondencia:

Carlos Girado-Polo, karlg21@hotmail.com

Recibido: 23 de enero de 2018

Aceptado: 24 de marzo de 2018

Editor: Germán Poveda 
cual es imposible calcular la ecuación correspondiente para la estimación de dicha variable, lo cual constituye una limitación de éstos.

Para resolver el problema descrito anteriormente, algunos autores han desarrollado modelos teóricos y semi-teóricos o híbridos utilizados en el cálculo de la radiación solar global de una atmósfera despejada, basados en las ecuaciones de transferencia radiativa de onda corta en la atmósfera (teoría de Lambert-Bouguer). En este sentido y como ejemplos, Brine e Iqbal (1983) desarrollaron un modelo espectral que permite estimar la radiación solar difusa y global, de una atmósfera despejada. En este modelo se consideran los procesos de extinción de la radiación solar en la atmósfera: absorción de la radiación solar por las moléculas de ozono, vapor de agua, los gases uniformemente mezclados y los aerosoles, y la dispersión de la radiación solar por efecto Rayleigh y Mie. Así mismo, Justus y Paris (1985) desarrollaron un modelo espectral utilizado en el cálculo de la radiación solar directa y difusa en la superficie terrestre, basados en aproximaciones similares a los modelos de Brine e Iqbal. Posteriormente, Bird y Riordan (1986), modificaron la componente de la radiación solar difusa de Justus y Paris (1985) mediante el algoritmo de transferencia radiativa BRITE. Por otra parte, Yang, et al. (2001) diseñaron un modelo para el cálculo de la radiación solar global de una atmósfera despejada, a partir de las componentes teóricas de las radiaciones directa y difusa (dispersión simple). Este modelo fue mejorado en Yang, et al. (2006) al desarrollar nuevas expresiones en el cálculo de las transmitancias atmosféricas, además de introducir en él datos de aerosoles y ozono disponibles a nivel mundial en vez de relaciones empíricas para el cálculo de estos. Posteriormente, Tang, et al. (2010) reescribieron las ecuaciones de transmitancia directa y difusa del modelo híbrido de Yang, et al. (2006), y a partir de éstas calcularon las componentes de la radiación solar global de una atmósfera despejada. Recientemente, Dai y Fang (2014) desarrollaron un algoritmo que mejoró la precisión del cálculo de la radiación solar global para una atmósfera despejada, a partir de una nueva expresión para la estimación de la radiación solar difusa.

En este trabajo se desarrolló un nuevo modelo híbrido para el cálculo de la radiación solar global de una atmósfera despejada, a partir de los valores espectrales de radiación solar directa y difusa (limitado a primera dispersión). En este modelo se propone y utiliza una nueva expresión teórica para la determinación de la fracción de la radiación solar difusa incidente sobre la superficie. El cálculo y validación del modelo desarrollado se realizó con datos de la estación Potsdam (Alemania) entre los años 2012 y 2014.

\section{Materiales y métodos}

El modelo desarrollado en el cálculo de la radiación solar, de una atmósfera despejada, está basado en la siguiente expresión espectral:

$$
K_{\lambda} \downarrow=K_{D \lambda} \downarrow+K_{d \lambda} \downarrow
$$

donde $K_{D \lambda} \downarrow$ es la radiación solar directa espectral y $K_{d \lambda} \downarrow$ es la radiación solar difusa espectral.

La radiación solar directa espectral incidente sobre la superficie $K_{D \lambda} \downarrow$ se determina a partir de la expresión de Bird y Riordan (1986):

$$
K_{D \lambda} \downarrow=K_{E \lambda}{ }^{*} \downarrow P_{\lambda}^{R} P_{\lambda}^{M} P_{\lambda}^{a} P_{\lambda}^{O_{3}} P_{\lambda}^{m g} P_{\lambda}^{H_{2} O}
$$

siendo, $K_{E \lambda}{ }^{*} \downarrow$ la radiación espectral extraterrestre efectiva, determinada por:

$$
K_{E \lambda}{ }^{*} \downarrow=K_{E \lambda} \downarrow f \cos \theta
$$

donde, $K_{E \lambda} \downarrow$ es la radiación espectral extraterrestre perpendicular o constante solar espectral, $f$ es el factor de corrección astronómica, ambos determinados por Fröhlich y London (1986), mientras que $\theta$ es el ángulo cenital solar. $P_{\lambda}^{R}$ y $P_{\lambda}^{M}$ son las transmitancias espectrales debidas a las dispersiones de Rayleigh y Mie respectivamente, y $P_{\lambda}^{a}, P_{\lambda}^{O_{3}}, P_{\lambda}^{m g}$ y $P_{\lambda}^{H_{2} \mathrm{O}}$ son las transmitancias espectrales debidas a la absorción de aerosoles, ozono, mezcla de gases (Justus y Paris, 1985) y vapor de agua, respectivamente.

La transmitancia espectral debida a la dispersión de Rayleigh $P_{\lambda}^{R}$, se determina por medio de

$$
P_{\lambda}^{R}=e^{-m_{r} \delta_{\lambda}^{R}}
$$

Donde $m_{r}$ es la masa relativa que se obtiene a partir de Kasten (1965)

$$
m_{r}=\frac{1}{\cos \theta+0,15(93,885-\theta)^{-1,253}}
$$

En la ecuación (4), $\delta_{\lambda}{ }^{R}$ es el coeficiente de dispersión adimensional de Rayleigh dado por Fröhlich y London (1986):

$$
\delta_{\lambda}^{R}=\left(\frac{p^{*}}{p_{0}}\right) 0,00865 \lambda^{-(3,916+0,074 \lambda+0,05 / \lambda)}
$$

siendo $\mathrm{p}_{0}$ la presión atmosférica al nivel del mar (1013.25 hPa) y $p^{*}$ es la presión atmosférica en el lugar de medición seleccionado.

La transmitancia espectral debida a la dispersión de Mie $P_{\lambda}^{M}$ tiene la forma:

$$
P_{\lambda}^{M}=e^{-m_{r} \delta_{\lambda}^{M}}
$$

en la cual el coeficiente de dispersión adimensional de Mie $\delta_{\lambda}{ }^{M}$ está dado por Ångström (1961):

$$
\delta_{\lambda}^{M}=\beta \lambda^{-1,3}
$$

$\beta$ es el coeficiente de turbidez de Ångström, determinado por medio del espesor óptico de los aerosoles AOD (Hess, et al., 1998)

$$
\beta=0,55^{1,3} \mathrm{AOD}(0,55)
$$

donde, $\operatorname{AOD}(0,55)$ es el espesor óptico debido a la dispersión de los aerosoles, normalizado a una longitud de onda de $0,55 \mu \mathrm{m}$.

La expresión para determinar la transmitancia espectral debido a la absorción de aerosoles $P_{\lambda}^{a}$, está dada por Russell, et al. (2010),

$$
P_{\lambda}^{a}=e^{-\left(1-\omega_{0 \lambda}\right) m_{r} \delta_{\lambda}^{M}}
$$

siendo, $\omega_{0 \lambda}$ el denominado albedo de dispersión simple. 
La transmitancia espectral debida a la absorción del

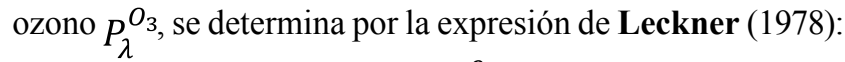

$$
P_{\lambda}^{O_{3}}=e^{-k_{\lambda}^{o_{3}} l m_{O_{3}}}
$$

$k_{\lambda}^{o_{3}}$ es el coeficiente de absorción del ozono, $l$ es el contenido de ozono en la atmósfera y $m_{O_{3}}$ es la masa relativa del ozono, que se obtiene mediante la ecuación de Iqbal (1983):

$$
m_{O_{3}}=\frac{1+z / R}{\left(\cos ^{2} \theta+2 z / R\right)^{1 / 2}}
$$

siendo, $\mathrm{z}$ la altitud del lugar de medida y $\mathrm{R}$ el radio de la Tierra.

De acuerdo a Leckner (1978), la transmitancia espectral debida a la mezcla de gases $P_{\lambda}^{m g}$ tiene la forma:

$$
P_{\lambda}^{m g}=e^{-1,41 k_{\lambda}^{m g} m_{a} /\left(1+118,93 k_{\lambda}^{m g} m_{a}\right)^{0.45}}
$$

donde, $k_{\lambda}^{m g}$ es el coeficiente de absorción de los gases uniformemente mezclados y $m_{a}$ es la masa relativa aparente, que se obtiene de la expresión de Kasten (1965):

$$
m_{a}=m_{r}\left(\frac{p^{*}}{p_{0}}\right)
$$

La transmitancia espectral debida a la absorción del vapor de agua $P_{\lambda}^{\mathrm{H}_{2} \mathrm{O}}$ se determina por Leckner (1978) y tiene la forma:

$$
P_{\lambda}^{\mathrm{H}_{2} \mathrm{O}}=e^{-0,2385 k_{\lambda}^{\mathrm{H}_{2} \mathrm{O}} m_{r} \omega /\left(1+20,7 k_{\lambda}^{\mathrm{H}_{2} \mathrm{O}} m_{r} \omega\right)^{0.45}}
$$

siendo, $k_{\lambda}^{\mathrm{H}_{2} \mathrm{O}}$ el coeficiente de absorción del vapor de agua y $\omega$ el contenido de agua precipitable en una columna de aire. Este último se determina así:

$$
\omega=0,493 \frac{H R}{100} \frac{p_{s}}{T}
$$

HR es la humedad relativa, T la temperatura del aire y $p_{s}$ la presión de saturación del vapor de agua, que se obtiene mediante la expresión de Leckner (1978), ecuación (17), en la cual $e^{26.23}$ contiene la dimensionalidad de esta presión:

$$
p_{s}=e^{26,23} e^{(-5416 / T)}
$$

Regresando a la ecuación (1), la estimación teórica de $K_{d \lambda} \downarrow$ es bastante compleja debido a las diferentes direcciones de la dispersión y a los respectivos estados de polarización de la radiación solar incidente. Como ilustración de esta situación Goody y Yung (1989) proponen una expresión para la determinación de la radiación solar dispersada por una atmósfera despejada en todas las direcciones, de la forma:

$$
\frac{\omega_{0 \lambda}}{4 \pi} \int_{4 \pi} \mathrm{I}_{\lambda} \downarrow \mathrm{F}_{\lambda}\left(\Omega, \Omega^{\prime}\right) \mathrm{d} \Omega^{\prime}
$$

donde, $I_{\lambda} \downarrow$ es la radiación solar incidente en una capa o volumen determinado de la atmósfera y $\mathrm{F}_{\lambda}\left(\Omega, \Omega^{\prime}\right)$ es la función de fase, dependiente de los ángulos sólidos de la radiación incidente $\Omega$ y dispersada $\Omega$ '. Puesto que en la práctica, la estimación de $F_{\lambda}$ (diferentes direcciones de dispersión y de polarización de la radiación solar incidente) presenta grandes dificultades, se recurrió a la ecuación propuesta por Justus y Paris (1985) en el cálculo de la radiación solar difusa espectral $K_{d \lambda} \downarrow$ :

$$
K_{d \lambda} \downarrow=K_{d \lambda}{ }^{R} \downarrow+K_{d \lambda}{ }^{M} \downarrow
$$

siendo, $K_{d \lambda}{ }^{R} \downarrow$ la radiación solar difusa debida a la dispersión de Rayleigh y $K_{d \lambda^{M}} \downarrow$ la debida a la dispersión de Mie. $K_{d \lambda^{R}} \downarrow$ está dado por:

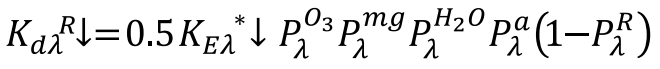

donde, 0,5 es la fracción de la radiación solar dispersada hacia la superficie terrestre por efecto Rayleigh.

Para la determinación de $K_{d \lambda}{ }^{M} \downarrow$ se utilizó la siguiente expresión teórica:

$$
K_{d \lambda}{ }^{M} \downarrow=f_{\lambda}^{M} K_{E \lambda}{ }^{*} \downarrow P_{\lambda}^{O_{3}} P_{\lambda}^{m g} P_{\lambda}^{H_{2} O} P_{\lambda}^{a}\left(1-P_{\lambda}^{M}\right)
$$

Siendo, $f_{\lambda}{ }^{M}$ la fracción de la radiación solar dispersada por los aerosoles hacia la superficie terrestre, la cual se determina a partir de la expresión teórica citada en Gónima (1993):

$$
f_{\lambda}^{M}=\frac{\omega_{0 \lambda}}{2}\left(1+g_{\lambda}\right)
$$

siendo, $g_{\lambda}$ el factor de asimetría.

Dada la importancia teórica de $f_{\lambda}^{M}$ en el cálculo de $K_{d \lambda}{ }^{M} \downarrow$ y para mejorar la descripción y precisión de la estimación de $K_{\lambda} \downarrow$ (ec. (1)), se modificó la ecuación (22), ya que esta describe únicamente la dispersión de la componente vertical de la radiación solar incidente sobre los aerosoles, como se muestra en la figura 1 para $\theta=0^{\circ}$.

En realidad, al incidir la radiación solar con diferentes ángulos $\theta$ (Figura 1), la radiación dispersada de Mie hacia un lugar determinado de la superficie terrestre varía a medida que $\theta$ aumenta. Por consiguiente y basado en Irvine (1963) esta dependencia se introdujo en la ecuación (22), de la siguiente forma:

$$
f_{\lambda}^{M}(\theta)=\frac{\omega_{0 \lambda}}{2}\left(1+g_{\lambda} \cos \theta\right)
$$

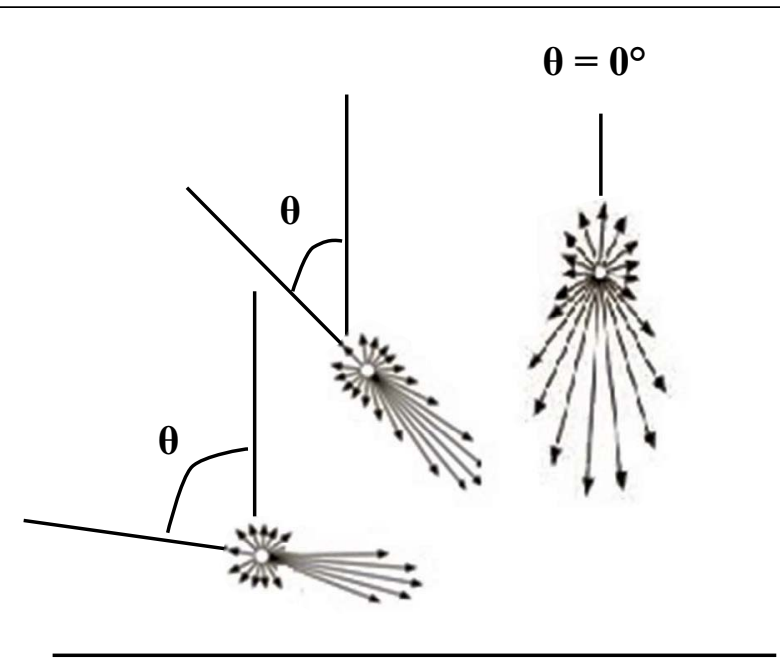

Superficie terrestre

Figura 1. Dispersión de Mie para diferentes ángulos cenitales $\theta$. 
Un análisis del comportamiento teórico de la ecuación (23) se realizó utilizando la ecuación (22) y el modelo validado y aceptado internacionalmente de Bird y Riordan, (1986). Estos últimos autores presentan una compleja expresión empírica para representar la dependencia directa de $f_{\lambda}^{M} \operatorname{con} \theta$.

El comportamiento de $f_{\lambda}^{M}(\theta)$, ec. (23), se muestra en la figura 2, comparado con el de los dos modelos mencionados anteriormente.

En la figura 2 se observa que $f_{\lambda}^{M}(\theta)$, ecuación (23), está por debajo de los valores de dicha fracción comparado con los de la ecuación (22) y de Bird y Riordan (1986). Sin embargo, para $\theta$ cercanos a la vertical $\left(\theta<10^{\circ}\right)$, los valores de $f_{\lambda}^{M}(\theta)$ estimados por la ecuación (23), son similares a los calculados con la ecuación (22) $\left(\theta=0^{\circ}\right)$. Para $\theta>10^{\circ}$, $f_{\lambda}^{M}(\theta)$ de la ecuación (23) muestra la dependencia con el ángulo, siendo esta mayor a medida que aumenta $\theta$. Respecto a Bird y Riordan (1986), a pesar de que su fracción también depende de $\theta$, los valores calculados con ésta están por encima de los obtenidos con las ecuaciones (22) y (23).

Continuando con el análisis de $f_{\lambda}^{M}(\theta)$, la figura 3 muestra el comportamiento espectral de $\operatorname{los} f_{\lambda}^{M}(\theta)$ de la ecuación (23) y de Bird y Riordan (1986), y el $f_{\lambda}^{M}\left(\theta=0^{\circ}\right)$ de la ecuación (22) para dos horas del día (ejemplo).

En la figura 3, se observa que los valores de $f_{\lambda}^{M}(\theta)$ de la ecuación (23) y de Bird y Riordan (1986) son mayores a las 13:00 horas comparados con los valores de la misma variable a las 6:00 hora local. Esto se debe a la dependencia de $f_{\lambda}^{M}(\theta)$ con el ángulo cenital (Figura 2) y por consiguiente la cantidad de radiación solar dispersada por los aerosoles hacia la superficie terrestre dependerá de la hora del día. Respecto a $f_{\lambda}^{M}\left(\theta=0^{\circ}\right)$ de la ecuación (22), tanto su comportamiento como sus valores en ambas horas del día son similares, es decir, los aerosoles dispersan porcentualmente la misma cantidad de radiación solar hacia la superficie terrestre a cualquier hora del día para una misma longitud de onda.

De acuerdo con lo anterior, la nueva expresión desarrollada para la determinación de $K_{d \lambda}{ }^{M} \downarrow$ fue:

$$
K_{d \lambda}{ }^{M} \downarrow=f_{\lambda}^{M}(\theta) K_{E \lambda}{ }^{*} \downarrow P_{\lambda}^{O_{3}} P_{\lambda}^{m g} P_{\lambda}^{H_{2} O} P_{\lambda}^{a}\left(1-P_{\lambda}^{M}\right)
$$

Una vez obtenidas todas las variables radiativas antes mencionadas, la radiación solar global integral $K \downarrow(0,3 \mu \mathrm{m}$ $-2,8 \mu \mathrm{m})$ se determinó así:

$$
K \downarrow=\int_{\lambda} K_{\lambda} \downarrow d \lambda=\int_{\lambda}\left(K_{D \lambda} \downarrow+K_{d \lambda} \downarrow\right) d \lambda=K_{D} \downarrow+K_{d} \downarrow
$$

siendo, $K_{D} \downarrow_{\mathrm{y}} K_{d} \downarrow$ las irradiancias solares directa y difusa integrales de una atmósfera despejada, respectivamente.

Los datos requeridos para el desarrollo del modelo fueron adquiridos a partir de las bases de datos meteorológicos y de radiación solar del servicio meteorológico alemán (Deutscher Wetterdienst, DWD, 2016), entre el periodo de tiempo comprendido entre el 01.01.2012 y el 31.12.2014, correspondientes a 17536 datos horarios seleccionados (debidamente validados) de la temperatura del aire $\mathrm{T}$, presión atmosférica $p$, humedad relativa HR, ángulo cenital solar $\theta$,

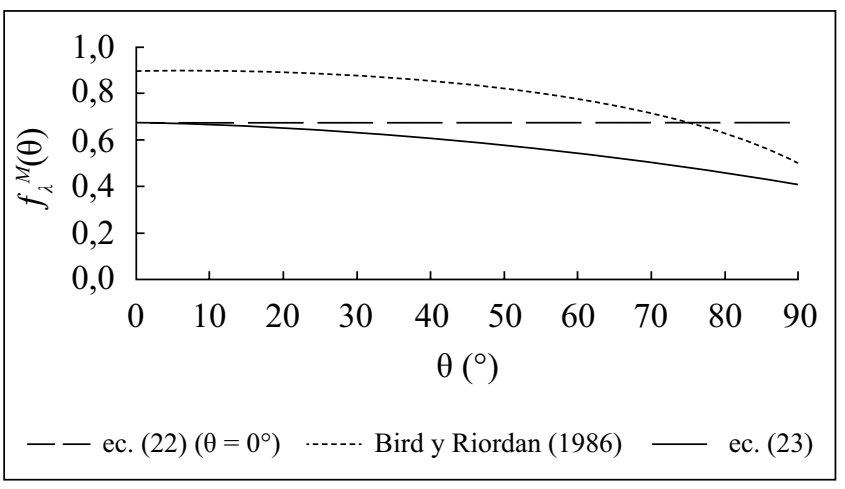

Figura 2. Variación de la fracción de la radiación solar dispersada por los aerosoles en dirección a la superficie terrestre $f_{\lambda}^{M}(\theta)$.

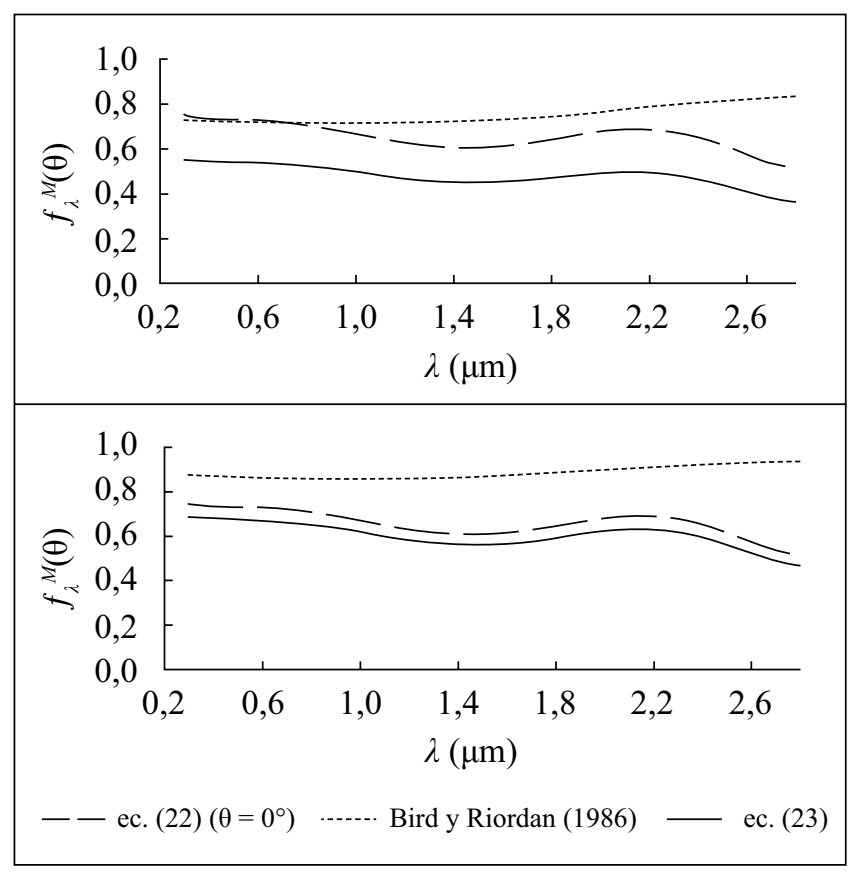

Figura 3. Variación de la fracción de la radiación solar dispersada por los aerosoles en dirección a la superficie terrestre $\left(f_{\lambda}^{M}(\theta)\right)$ en función de $\lambda$, correspondiente al día 25 de julio de 2012 a las (a) 6:00 horas local $\operatorname{con} \theta=37.25^{\circ}$ y (b) $13: 00$ horas local $\operatorname{con} \theta=70.11^{\circ}$.

radiación solar global $K \downarrow$ y complementariamente la fracción del brillo solar. La estación meteorológica y radiométrica seleccionada está localizada en Potsdam-Alemania (52 $22^{\prime} 48^{\prime \prime} \mathrm{N}, 13^{\circ} 3^{\prime} 36^{\prime \prime}$ E y $\left.81 \mathrm{msnm}\right)$. Del total de los datos antes mencionados para cada variable, se seleccionaron los correspondientes a una atmósfera despejada, utilizando la fracción del brillo solar en la identificación de éstos. A continuación, se eliminaron aquellos datos que correspondían a HR mayores al 90\% (atmósfera con un alto contenido de vapor de agua), lo que en la práctica no representa las condiciones de una atmósfera despejada. Posteriormente, se descartaron aquellos datos de las fechas, en las cuales no existían las correspondientes mediciones de ozono de acuerdo a NASA 
(2015). Finalmente, se obtuvieron 1350 datos horarios de T, $p$, HR, $\theta$ y $K \downarrow$ de una atmósfera despejada, correspondientes a 315 días calendario, con los cuales se procedió al cálculo de la radiación solar global integral, ecuación (25).

Para el cálculo de $K_{\lambda} \downarrow$ en la ecuación (1), fue necesario determinar inicialmente $K_{D \lambda} \downarrow$ por medio de la ecuación (2). Para esto se calculó $K_{E \lambda}{ }^{*} \downarrow$, ecuación (3), a partir de la constante solar espectral $K_{E \lambda} \downarrow$ y del factor de corrección astronómica f (Fröhlich y London, 1986), así como del ángulo cenital $\theta$. Posteriormente, se determinaron las transmitancias atmosféricas espectrales en su orden:

a) Se calculó $P_{\lambda}^{R}$, ecuación (4), por medio de $m_{r}$, ecuación (5) y $\delta_{\lambda} R$,ecuación (6).

b) Se determinó $P_{\lambda}^{M}$, ec. (7), en la cual $\delta_{\lambda}{ }^{R}$ y $\beta$ se calcularon mediante las ecuaciones (8) y (9), respectivamente. En la determinación del AOD, se utilizó el software Optical Properties of Aerosols and Clouds OPAC (OPAC, 2016), que además de calcular el AOD, proporciona el contenido y las propiedades ópticas de los aerosoles de la atmósfera para diferentes longitudes de onda y diferentes valores de HR. El procedimiento utilizado en el cálculo del AOD fue el siguiente:

Inicialmente se calculó esta variable utilizando solamente el modelo atmosférico Continental Polluted para los aerosoles, descrito en el software mencionado.

Debido a que la estación meteorológica y radiométrica en Potsdam, situada al suroeste de Berlín, está en las cercanías de esta última (35 km aproximadamente), su atmósfera está fuertemente influenciada por la de Berlín (ciudad industrial), la cual permanece parte del año afectada por contaminantes atmosféricos en forma de aerosoles generados por la actividad industrial y los sistemas de calefacción de carbón y kerosene (Alemania guía de vivienda, 2016). En este sentido y puesto que la atmósfera de interés presentó marcadas variaciones durante el transcurso del tiempo, debidas al contenido de aerosoles, como se pudo comprobar posteriormente, al analizar los resultados calculados de la radiación solar global utilizando un solo tipo de atmósfera (Continental Polluted del OPAC), fue necesario seleccionar cuatro tipos de atmósfera, representativas del contenido y propiedades ópticas de los aerosoles existentes en la atmósfera sobre el lugar de medición (Urban-UR, Continental Polluted-CP, Continental Average-CA y Continental Clean-CC). Estos modelos de atmósferas fueron aplicados selectivamente en el cálculo de los datos horarios del AOD. En el OPAC, para el cálculo del AOD, se utiliza HR como variable representativa de las condiciones termodinámicas de la atmósfera existentes sobre el lugar de medición, cuyos valores nominales son $0 \%, 50 \%$, $70 \%, 80 \%, 90 \%, 95 \%, 98 \%$ y $99 \%$ (OPAC, 2016). Entonces, para la determinación de valores horarios de AOD (datos de HR diferentes a los nominales), fue necesario calcular y aplicar una correlación estadística entre los AOD calculados para cada uno de los datos nominales de HR y éstos.

c) Se estimó $P_{\lambda}^{a}$ utilizando la ecuación (10), en la cual para determinar el albedo de dispersión simple $\omega_{0 \lambda}$, se seleccionaron los datos discretos existentes de $\omega_{0 \lambda}$ (WCP, 1986), con los cuales se calculó una regresión estadística $\left(\omega_{0 \lambda}, \lambda\right)$ para con ella calcular $\omega_{0 \lambda}$ en el rango espectral $0,3 \mu \mathrm{m}-2,8 \mu \mathrm{m}$.

d) Se calculó $P_{\lambda}^{O_{3}}$ mediante la ecuación (11). Los valores de $k_{\lambda}^{o_{3}}$ requeridos se adquirieron de las tablas existentes en Leckner (1978). Los valores de $l$ (contenido de $\mathrm{O}_{3}$ ) se extrajeron de las bases de datos específicas de la NASA (2015), correspondientes a las fechas de los 1350 datos seleccionados como representativos de una atmósfera despejada. Finalmente, $m_{\mathrm{O}_{3}}$ se estimó a partir de la ecuación (12).

e) Se determinó $P_{\lambda}^{m g}$ utilizando la ecuación (13), en la cual los valores de $k_{\lambda}^{m g}$ requeridos se adquirieron de las tablas existentes en Leckner (1978) y $m_{a}$ se estimó a partir de la ecuación (14).

f) Se estimó $P_{\lambda}^{\mathrm{H}_{2} \mathrm{O}}$ a partir de la ecuación (15), en la cual los valores de $k_{\lambda}^{\mathrm{H}_{2} \mathrm{O}}$ se adquirieron de las tablas existentes en Leckner (1978). $\omega$ se determinó mediante la ecuación. (16) y $p_{s}$ mediante de la ecuación (17).

La componente de la radiación solar difusa espectral $K_{d \lambda} \downarrow$, ecuación (19), se calculó de la siguiente forma:

a) $K_{d \lambda}{ }^{R} \downarrow$ se calculó a partir de la ecuación (20).

b) $K_{d \lambda^{M}}{ }^{M}$, ec. (24), en la cual $f_{\lambda}^{M}(\theta)$ se determinó mediante la ecuación (23). Para calcular el factor de asimetría $g$, se seleccionaron los datos discretos existentes de $g$ (WCP, 1986), a partir de los cuales se calculó una regresión estadística $(g, \lambda)$ para con ella estimar esta variable entre $0,3 \mu \mathrm{m}$ y $2,8 \mu \mathrm{m}$.

Una vez realizado lo anterior, la radiación solar global integral $K \downarrow$ para los 1350 datos horarios seleccionados correspondientes a una atmósfera despejada, se determinó a partir de la ecuación (25). Finalmente, se validaron los resultados de $K \downarrow$, calculados por medio del modelo desarrollado, con las mediciones hechas en campo de esta misma variable en la estación meteorológica Potsdam, (01.01.2012 - 31.12.2014), comparándolos al mismo tiempo con los resultados de $K \downarrow$ obtenidos utilizando otros tres modelos seleccionados de la literatura especializada actual: Yang, et al. (2006), Tang, et al. (2010), y Dai y Fang (2014).

\section{Resultados y discusión}

El modelo desarrollado de una atmósfera despejada, permite hacer cálculos horarios de la radiación solar global, a partir de variables meteorológicas que describen el estado de la atmósfera y de otras variables radiativas que requieren ser calculadas. En análisis de los resultados obtenidos se escogió un día en particular ( 25 de julio de 2012), ya que para el resto de días seleccionados con atmósfera despejada (315 en total), el comportamiento mostrado por las variables involucradas es similar.

Dada la importancia que tiene la radiación solar difusa en el cálculo de la radiación solar global, inicialmente se hace un análisis exhaustivo de dicha variable en función de la radiación solar dispersada hacia la superficie terrestre por los aerosoles, al igual que el tipo de atmósfera seleccionado. 
Finalmente, se comparan los resultados de $K \downarrow$ calculados con el nuevo modelo y los obtenidos aplicando los otros tres modelos mencionados anteriormente.

Al realizar inicialmente los cálculos de $K_{\lambda} \downarrow$ con un solo modelo atmosférico (CP) se obtuvieron resultados que difirieron en gran medida respecto a los datos medidos de esta variable, y puesto que la ubicación de la zona de estudio está influenciada por los aerosoles provenientes de la ciudad de Berlín, fue necesario utilizar los cuatro modelos atmosféricos definidos anteriormente, para mejorar la precisión del cálculo de $K_{\lambda} \downarrow$ (ec. (1)). Las características de los aerosoles que componen cada una de las atmósferas seleccionadas, para diferentes valores de HR, se muestran en la tabla 1S, https://www.raccefyn.co/index.php/raccefyn/ article/downloadSuppFile/610/2579 (Hess, et al., 1998).

Analizando los datos de la composición de las cuatro atmósferas seleccionadas (Tabla 1S), $\rho$ es diferente en las tres componentes (Partículas insolubles en agua, Partículas solubles en agua y Hollín) de cada una de las atmósferas seleccionadas. En particular, la componente insoluble muestra una mayor densidad respecto a las otras dos componentes. Respecto a N, para las componentes Partículas insolubles en agua, Partículas solubles en agua y Hollín, UR tiene un mayor número de partículas que en las otras tres atmósferas. Por otra parte, también se observa que HR es la causa de la variación de $\rho$ para la componente Partículas solubles en agua (Figura 4), mientras que en las otras dos componentes HR no ejerce ninguna influencia sobre $\rho$.

La disminución de $\rho$ con el aumento de HR (Figura 4) se debe a que la componente Partículas solubles en agua, como era de esperarse, experimenta una mayor disolución con el aumento de HR, disminuyendo la cantidad de éstas que ocupaban el volumen inicial.

De acuerdo con OPAC, para el cálculo del AOD son necesarios los valores de las componentes (Partículas insolubles en agua, Partículas solubles en agua y Hollín) mostradas en la tabla $1 \mathrm{~S}$. Como se muestra en la tabla $1 \mathrm{~S}$, las componentes Partículas insolubles en agua y Hollín no dependen de HR, mientras que $\rho$ de Partículas solubles en agua, como se mencionó anteriormente, varía en función de esta variable meteorológica.

La figura 5 muestra el comportamiento del AOD en función de $\rho$ de Partículas solubles en agua.

En la figura 5, el AOD de las cuatro atmósferas seleccionadas disminuye con el incremento de $\rho$. Este comportamiento del AOD, que está influenciado por la componente Partículas solubles en agua, es consecuencia de la disolución de las partículas de aerosol en el agua existente en la atmósfera (en un momento determinado), aumentando la densidad del nuevo compuesto y disminuyendo al mismo tiempo la capacidad de éste para dispersar la radiación solar incidente, como resultado de una mayor absorción de ésta. Por otra parte, UR es la atmósfera que muestra mayores valores de AOD respecto a las otras dos, debido a que ésta es la que contiene mayor número de partículas de las tres componentes (Tabla 1S).

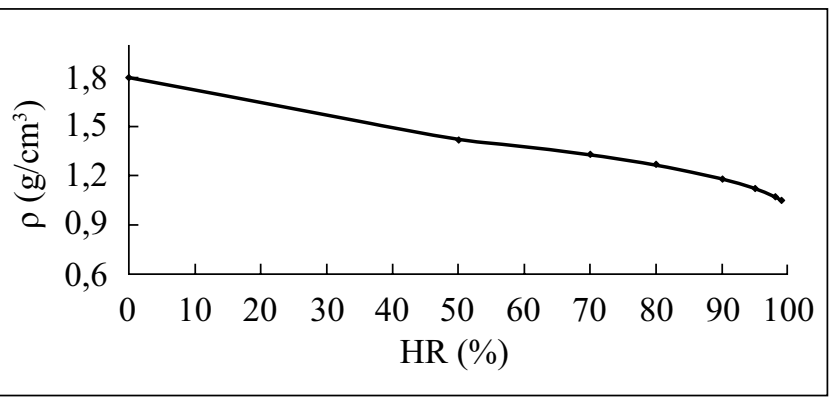

Figura 4. Comportamiento de $\rho$ de la componente Partículas solubles en agua con la variación de HR.

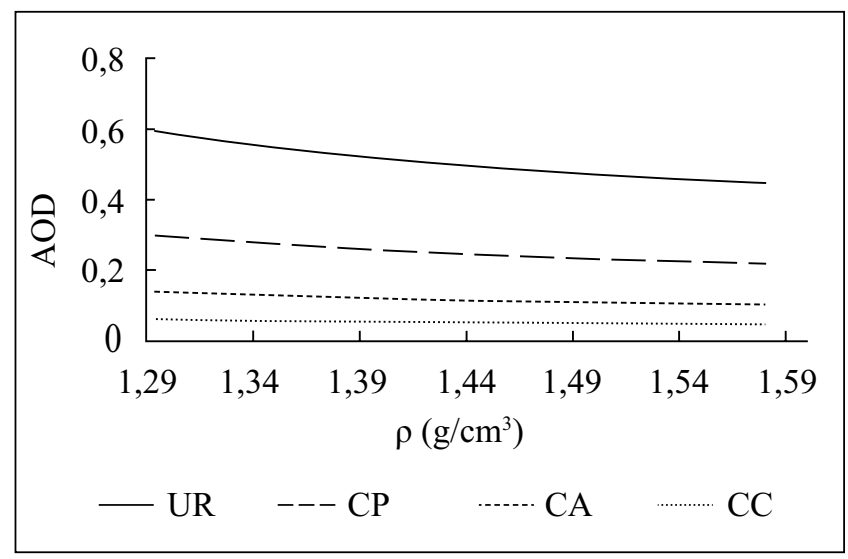

Figura 5. Comportamiento del AOD en función de $\rho$ de la componente Partículas solubles en agua, para las cuatro atmósferas seleccionadas.

Debido a la importancia del AOD en la determinación del coeficiente de dispersión de Mie (ecuaciones (8) y (9)), las transmitancias atmosféricas por dispersión de Mie (ecuación (7)) y absorción de aerosoles (ecuación (10)), a continuación se hace un análisis del comportamiento de estas variables radiativas en los diferentes tipos de atmósferas.

En la figura 1S, https:/www.raccefyn.co/index.php/ raccefyn/article/downloadSuppFile/610/2572, la componente Partículas solubles en agua muestra valores más altos del coeficiente $\delta_{\lambda}^{M}$ para la atmósfera UR respecto a las otras tres atmósferas. Asimismo, se observa que este coeficiente disminuye con el incremento de $\rho$, siendo este comportamiento similar al del AOD, débilmente modificado por $\lambda^{-1.3}$ (ecuación (8)). Además y de acuerdo con la figura $2 \mathrm{~S}$, https:// www.raccefyn.co/index.php/raccefyn/article/download SuppFile/610/2573, se observa que la mayor dispersión de la radiación solar incidente en las cuatro atmósferas seleccionadas se efectúa entre $0,3 \mu \mathrm{m}$ y $1,0 \mu \mathrm{m}$, mientras que para valores $>1 \mu \mathrm{m}, \delta_{\lambda}^{M}$ disminuye continuamente a medida que aumenta $\lambda$. Este comportamiento de $\delta_{\lambda}^{M}$ concuerda con la teoría (Goody y Yung, 1989), siendo UR la atmósfera con mayor capacidad de dispersión por su mayor densidad de partículas. 
Para analizar el comportamiento de la transmitancia por dispersión de Mie y puesto que según la ecuación (7) ésta depende de $\delta_{\lambda}^{M}$, coeficiente que a la vez está relacionado con el contenido de vapor de agua en la atmósfera (Figura $1 \mathrm{~S})$, es necesario analizar la dependencia entre esta transmitancia y $\rho$ de Partículas solubles en agua, correspondiente a los modelos de las cuatro atmósferas seleccionadas. En la figura 3S, https://www.raccefyn.co/index.php/raccefyn/ article/downloadSuppFile/610/2574, se observa que, en las cuatro clases de atmósfera, $P_{\lambda}^{M}$ aumenta con el incremento de $\rho$ entre $1,29 \mathrm{~g} / \mathrm{cm}^{3}$ y $1,58 \mathrm{~g} / \mathrm{cm}^{3}$, mientras que a partir de $1,58 \mathrm{~g} / \mathrm{cm}^{3}$ (16:00 horas) estas dos variables disminuyen. De acuerdo con la ecuación (7), el aumento (6:00 horas a 15:00 horas) y posterior disminución ( $>16$ horas) de $P_{\lambda}^{M}$ se debe en parte al comportamiento mostrado por $\delta_{\lambda}^{M}$ (Figura 4S a, https://www.raccefyn.co/index.php/raccefyn/ article/downloadSuppFile/610/2575), pero especialmente al aumento abrupto de $\mathrm{m}_{\mathrm{r}}$ a partir de las 16:00 horas (Figura $4 \mathrm{~S} b$ ). Este comportamiento abrupto de $\mathrm{m}_{\mathrm{r}}$ posiblemente pudo haberse debido a una mayor emisión de gases industriales en la ciudad de Berlín, que eventualmente pudieron ser trasladados a la ciudad de Potsdam por efecto de los vientos, modificando su atmósfera.

De acuerdo con la figura 5S, https://www.raccefyn.co/ index.php/raccefyn/article/downloadSuppFile/610/2576, y en concordancia con la teoría, $P_{\lambda}^{M}$ es mayor entre $0,3 \mu \mathrm{m}$ y $1,0 \mu \mathrm{m}$, mientras que para $\lambda>1 \mu \mathrm{m}$ ésta es mucho menor. Además, la atmósfera UR presenta menor transmitancia comparada con las otras tres atmósferas, debido a que la concentración de aerosoles y por ende la extinción de la radiación solar por dispersión por parte de éstos es mayor comparada con la de las otras tres.

Finalmente, en la figura 6S, https://www.raccefyn.co/ index.php/raccefyn/article/downloadSuppFile/610/2577, se muestra el comportamiento espectral por absorción de aerosoles en la atmósfera. En esta figura se observa que en las cuatro atmósferas, los aerosoles absorben mayor cantidad de radiación solar entre $0,3 \mu \mathrm{m}$ y 0,7 aproximadamente, debido a que en este rango espectral y de acuerdo con la ecuación (10), los valores de $\delta_{\lambda}^{M}$ son mayores (Figura 2S) que la cantidad $\left(1-\omega_{0 \lambda}\right)$, representativa de la radiación absorbida por los aerosoles mientras que a partir de $0,7 \mu \mathrm{m}$ la absorción disminuye.

Puesto que $K_{d \lambda}{ }^{M} \downarrow$ (ecuación (24)) depende de de la fracción de la radiación solar dispersada por los aerosoles hacia la superficie terrestre, la figura 6 muestra la variación espectral de $K_{d \lambda}{ }^{M} \downarrow$ calculada para un día y horas particulares (por ejemplo), utilizando los valores de dicha fracción (Figura 2).

En la figura 6 se observa que los valores $K_{d \lambda}{ }^{M} \downarrow$ para los tres modelos de la fracción de dispersión (ecuación (22), Bird y Riordan (1986) y ecuación (23)) son mayores a las 13:00 hora local que a las 6:00 horas, esto debido a la dependencia de esta fracción con el ángulo cenital (Figura 2). En el cálculo de $K_{d \lambda}{ }^{M} \downarrow$ en el nuevo modelo se utilizó la estimada a partir de $f_{\lambda}^{M}(\theta)$ obtenida mediante la ecuación (23).
La otra componente de la radiación solar global, representada por $K_{D \lambda} \downarrow$ fue determinada a partir de las transmitancias espectrales $\left(P_{\lambda}^{R}, P_{\lambda}^{M}, P_{\lambda}^{a}, P_{\lambda}^{m g}, P_{\lambda}^{H_{2} O}\right.$ y $\left.P_{\lambda}^{O_{3}}\right)$, se muestra en la figura $7 \mathrm{~S}$, https://www.raccefyn.co/index. php/raccefyn/article/downloadSuppFile/610/2578. En esta figura se confirma el hecho de que $K_{D \lambda} \downarrow$ se extingue en mayor magnitud cuando atraviesa una mayor masa atmosférica (6:00 horas), con respecto a la menor extinción de $K_{D \lambda} \downarrow$ a las 13:00 horas. Espectralmente (Figura 7S), $K_{E \lambda}{ }^{*} \downarrow$ y $K_{D \lambda} \downarrow$ muestran el típico comportamiento de la radiación solar tanto en el límite superior de la atmósfera (aproximadamente cuerpo negro) como en la superficie terrestre (dispersión y absorción).

Es así como, basándose en la ecuación (25), se compararon inicialmente los valores horarios calculados de $K \downarrow$ con los respectivos valores medidos en Potsdam (DWD, 2016) y posteriormente con los resultados obtenidos de la misma variable mediante la aplicación de otros tres modelos seleccionados de la literatura especializada actual (Yang, et al. (2006), Tang, et al. (2010) y Dai y Fang (2014)).

Para examinar la relación entre los $K_{\lambda} \downarrow$ medidos y calculados con los diferentes modelos, se realizó inicialmente un análisis de regresión estadística (Figura 7), en el cual se utilizó el coeficiente de correlación $r$ para analizar dicha relación. Además, otros criterios estadísticos calculados y utilizados en este análisis fueron rangos y promedios de las diferencias entre los valores medidos y calculados, el error cuadrático medio (ECM) y el número de datos de $K_{\lambda} \downarrow$ calculados que difieren de los medidos en una cantidad menor al $10 \%$ y al $5 \%$ o precisión de la estimación de dicha variable con el nuevo modelo desarrollado (Tabla 1).

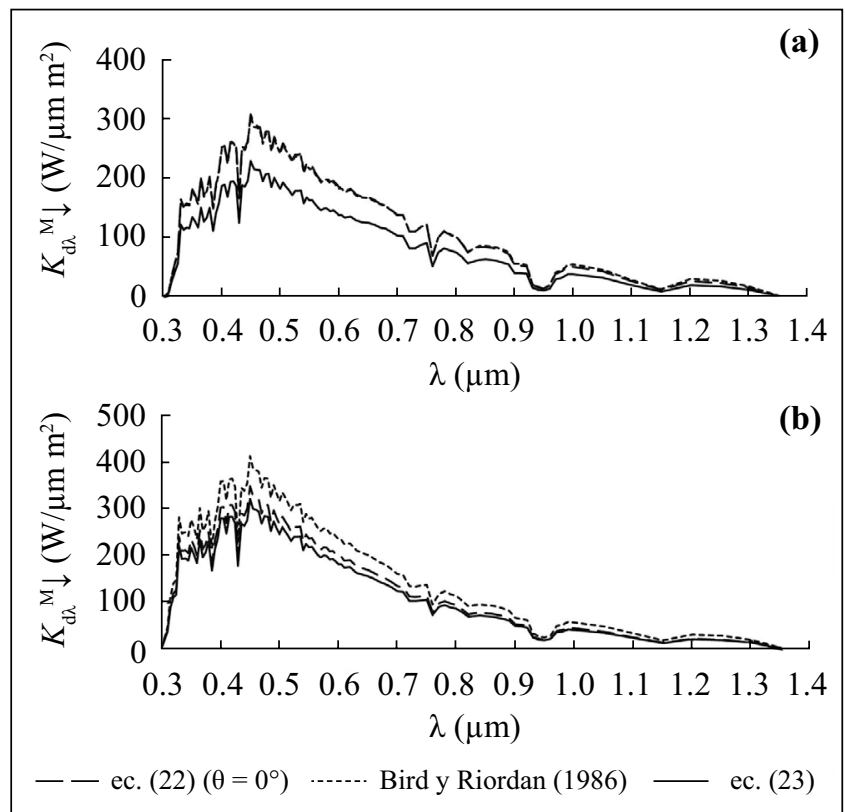

Figura 6. Variación de la radiación solar difusa espectral por dispersión de Mie $\left(K_{d \lambda}{ }^{M} \downarrow\right)$ en función de $\lambda$, correspondiente al día 25 de julio de 2012 a las (a) 6:00 horas local y (b) 13:00 horas local. 


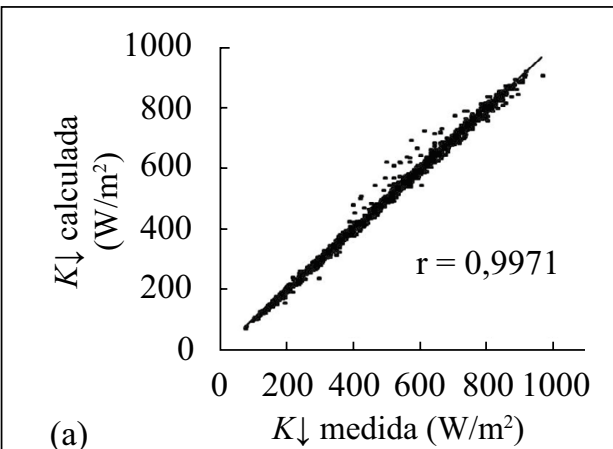

(a)

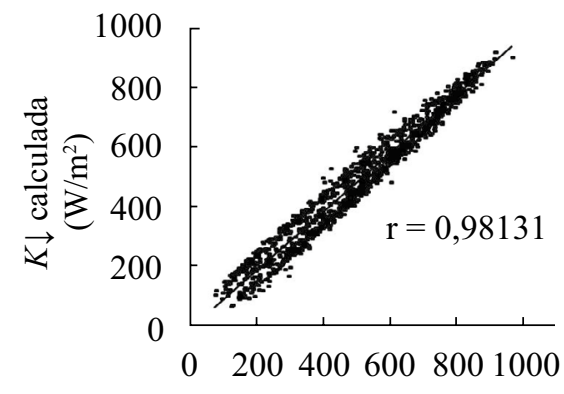

(c)
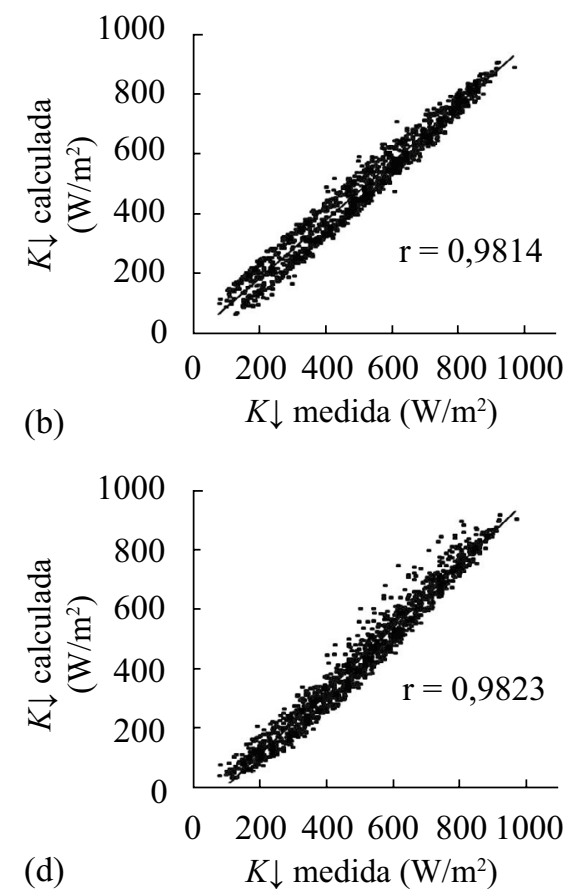

(b) Yang, et al. (2006)

(d) Dai y Fang (2014)

(a) Nuevo modelo

(c) Tang, et al. (2010)

Figura 7. Valores de radiación solar global medida y calculada en la ciudad de Potsdam durante los periodos del 2012 al 2014, correspondientes a una atmósfera despejada.

Tabla 1. Comparación estadística de los datos de $K \downarrow$ calculados con los diferentes modelos (Número total de datos de atmósfera despejada: 1350).

\begin{tabular}{lcccc}
\hline & $\begin{array}{c}\boldsymbol{K} \downarrow \\
\text { (nuevo modelo) }\end{array}$ & $\begin{array}{c}\boldsymbol{K} \downarrow \\
\text { (Yang, } \text { et al., 2006) }\end{array}$ & $\begin{array}{c}\boldsymbol{K} \downarrow \\
\text { (Tang, } \text { et al., 2010) }\end{array}$ & $\begin{array}{c}\boldsymbol{K} \downarrow \\
\text { (Dai y Fang, 2014) }\end{array}$ \\
\hline$\Delta\left(\mathbf{W} / \mathbf{m}^{2}\right)$ & $-124-60$ & $-108-125$ & $-101-126$ & $-133-158$ \\
$\bar{\Delta}\left(\mathbf{W} / \mathbf{m}^{2}\right)$ & $-1,42$ & 17,88 & 22,38 & 66,05 \\
$\mathbf{E C M}\left(\mathbf{W} / \mathbf{m}^{2}\right)$ & 15,75 & 45,61 & 43,72 & 79,14 \\
Número de datos $<\mathbf{1 0} \%$ de precisión & 1329 & 780 & 800 & 497 \\
Número de datos $<\mathbf{5} \%$ de precisión & 1265 & 446 & 461 & 274 \\
\hline
\end{tabular}

$\Delta$ : rango de las diferencias entre los datos medidos y calculados; $\bar{\Delta}$ : promedio de las diferencias entre los valores medidos y calculados.

En la figura 7 se observa que la dispersión de los datos es menor en el nuevo modelo desarrollado, comparado con la de los otros tres modelos, lo que indica que el nuevo modelo presenta un mejor ajuste estadístico, como se demuestra con los correspondientes r. Continuando con el análisis de $K \downarrow$, la tabla 1 muestra el resto de los criterios estadísticos utilizados para su análisis.

En la tabla 1 se observa que los $\Delta$ y $\bar{\Delta}$ calculados con el nuevo modelo desarrollado, son menores comparado con los otros tres modelos. Además, al comparar los ECM, se observa que el nuevo modelo tiene el valor más bajo respecto a los demás modelos. Por otra parte, teniendo en cuenta el número de datos de $K \downarrow$ calculados $<10 \% \mathrm{y}<5 \%$ de precisión, el nuevo modelo desarrollado muestra una mayor precisión del cálculo de $K \downarrow$ que los otros modelos.

De acuerdo con el análisis estadístico anterior, el nuevo modelo desarrollado representa con mayor precisión las correspondientes mediciones de $K \downarrow$, comparado con los otros tres modelos seleccionados.

\section{Conclusiones}

Al comparar los criterios estadísticos utilizados para el análisis entre los valores de radiación solar global medidas y calculadas por los diferentes modelos, el nuevo modelo 
desarrollado presenta un coeficiente de correlación $\mathrm{r}$ igual a 0,9971, mientras que los modelos de Yang, et al. (2006), Tang, et al. (2010) y Dai y Fang (2014) presentan coeficientes iguales a $0,9814,0,9813$ y 0,9823 , respectivamente. Además, el rango de las diferencias entre los datos medidos y calculados son menores en el nuevo modelo desarrollado comparado con los tres modelos restantes. Por otra parte, el nuevo modelo desarrollado presenta un error cuadrático medio ECM igual a $15,75 \mathrm{~W} / \mathrm{m}^{2}$, Yang, et al. (2006) de $45,61 \mathrm{~W} / \mathrm{m}^{2}$, Tang, et al. (2010) de 43,72 W/m² y Dai y Fang (2014) de 79,14 W/m². Por último, el $98 \%$ de los datos de la radiación solar global $K \downarrow$, calculados con el nuevo modelo desarrollado, están por debajo del $10 \%$ de precisión y el $94 \%$ de éstos son menores que el $5 \%$ de precisión, mientras que para los otros tres modelos la concordancia es inferior al $62 \%$ para $10 \%$ y $32 \%$ para $5 \%$ de precisión. Por consiguiente, el nuevo modelo desarrollado permite obtener resultados más precisos de la radiación solar global $K \downarrow$ horaria

La precisión del cálculo de la radiación solar global, depende estrechamente de una mejor determinación de la radiación solar difusa por dispersión de Mie, la cual se logró introduciendo la variación de la fracción de dispersión $f_{\lambda}^{M}(\theta)$ con el ángulo de incidencia de la radiación solar $\theta$.

Otras diferencias entre el nuevo modelo desarrollado y los modelos de Yang, et al. (2006), Tang, et al. (2010) y Dai y Fang (2014) fueron que el cálculo de todas las variables radiativas se realizó en forma espectral y que además se incluyó la transmitancia de los aerosoles por absorción.

En el nuevo modelo se consideran todos los procesos de extinción radiativa en la atmósfera, al igual que los efectos de los aerosoles, mezcla de gases, vapor de agua y el ozono, lo que permite realizar cálculos de la radiación solar global horaria de una atmósfera despejada, a partir de la temperatura del aire, la humedad relativa, la presión atmosférica, el AOD, el contenido de ozono, mezcla de gases y vapor de agua.

De acuerdo con que el nuevo modelo desarrollado está estructurado teóricamente, es posible utilizarlo en el cálculo horario de la radiación solar global de una atmósfera despejada en cualquier región del mundo, siempre que se disponga de datos de las variables meteorológicas (temperatura del aire, humedad relativa y presión atmosférica), radiativas (constante solar espectral, albedo de dispersión simple, factor de asimetría y coeficientes de absorción del $\mathrm{O}_{3}, \mathrm{mg}$ y $\mathrm{H}_{2} \mathrm{O}$ ) y del contenido de ozono de la atmósfera.

Debido a que el contenido de aerosoles atmosféricos sobre la ciudad de Potsdam está influenciado por el de Berlín y la importancia que este tiene sobre la radiación solar incidente, se consideraron cuatro modelos atmosféricos (aerosoles) para el cálculo de la radiación solar global $K \downarrow$, mejorando consecuentemente la precisión en los cálculos de esta variable radiativa.

\section{Conflicto de intereses}

Los autores declaran no tener conflicto de intereses.

\section{Contribución de los autores}

Trabajo de tesis de maestría de Carlos Giraldo Polo bajo la dirección de Leonardo Gónima Gónima.

\section{Información Suplementaria}

Tabla 1S. Descripción de las características de las atmósferas seleccionadas (UR, CP, CA y CC) para diferentes valores de HR (Hess, et al., 1998). Vea la tabla 1S en: https://www.raccefyn.co/index.php/raccefyn/article/ downloadSuppFile/610/2579

Figura 1S. Comportamiento de $\delta_{\lambda}^{M}$ en función de $\rho$ de la componente Partículas solubles en agua, para $\lambda=1 \mu \mathrm{m}$ por ejemplo, correspondiente al día 25 de julio de 2012, entre las 6:00 horas y las 18:00 horas locales. Vea la figura 1S en: https://www.raccefyn.co/index.php/raccefyn/article/ downloadSuppFile/610/2572

Figura 2S. Comportamiento espectral de $\delta_{\lambda}^{M}: 25$ de julio de 2012 a las 6:00 hora local $\left(\mathrm{T}=18.4^{\circ} \mathrm{C}, \mathrm{HR}=75 \%\right)$. Vea la figura $2 \mathrm{~S}$ en: https:/www.raccefyn.co/index.php/raccefyn/ article/downloadSuppFile/610/2573

Figura 3S. Variación de $P_{\lambda}^{M}$ en función de $\rho$ de la componente Partículas solubles en agua, para $\lambda=1 \mu \mathrm{m}$ por ejemplo, correspondiente al día 25 de julio de 2012, entre las 6:00 horas y las 18:00 horas locales. Vea la figura $3 \mathrm{~S}$ en: https://www.raccefyn.co/index.php/raccefyn/article/ downloadSuppFile/610/2574

Figura 4S. Variación temporal de (a) $\delta_{\lambda}^{M}$ para $\lambda=1 \mu \mathrm{m}$ (ejemplo) y (b) $\mathrm{m}_{\mathrm{r}}$, correspondiente al día 25 de julio de 2012, entre las 6:00 horas y las 18:00 horas locales. Atmósfera UR por ejemplo. Vea la figura $4 \mathrm{~S}$ en: https://www.raccefyn. co/index.php/raccefyn/article/downloadSuppFile/610/2575

Figura 5S. Comportamiento de la transmitancia atmosférica espectral por dispersión de aerosoles calculada $P_{\lambda}^{M}: 25$ de julio de 2012 a las 6:00 hora local $\left(\mathrm{T}=18.4^{\circ} \mathrm{C}, \mathrm{HR}=75 \%\right)$. Vea la figura $5 \mathrm{~S}$ en: https://www.raccefyn.co/index.php/ raccefyn/article/downloadSuppFile/610/2576

Figura 6S. Variación espectral de la transmitancia atmosférica calculada por la absorción de los aerosoles $\left(P_{\lambda}^{a}\right): 25$ de julio de 2012 a las 6:00 hora local $\left(\mathrm{T}=18.4{ }^{\circ} \mathrm{C}, \mathrm{HR}=75 \%\right)$. Vea la figura $6 \mathrm{~S}$ en: https://www.raccefyn.co/index.php/ raccefyn/article/downloadSuppFile/610/2577

Figura 7S. Variación de la radiación solar espectral directa $\left(K_{D \lambda} \downarrow\right)$ y extraterrestre efectiva $\left(K_{E \lambda}{ }^{*} \downarrow\right)$ en función de $\lambda$, correspondiente al día 25 de julio de 2012 a las (a) 6:00 hora local $\left(\mathrm{T}=18.4{ }^{\circ} \mathrm{C}, \mathrm{HR}=75 \%\right)$ y (b) 13:00 hora local $\left(\mathrm{T}=31,1{ }^{\circ} \mathrm{C}, \mathrm{HR}=42 \%\right)$. Vea la figura $7 \mathrm{~S}$ en: https:// www.raccefyn.co/index.php/raccefyn/article/download SuppFile/610/2578

\section{Referencias}

Alemania guía de vivienda. (2016). Disponible en https:// www.justlanded.com/espanol/Alemania/Guia-Alemania/ Alojamiento/Vivienda, accedida en marzo de 2016. 
Ångström, A. (1924). Solar and terrestrial radiation. Quarterly Journal of the Royal Meteorological Society. 50: 121-126.

Ångström, A. (1961). Techniques of determining the turbidity of the atmosphere. Tellus, 13: 214-223.

Bird, R., Riordan, C. (1986). Simple solar spectral model for direct and diffuse irradiance on horizontal and tilted planes at the Earth's surface for cloudless atmospheres. J. Climate Appl. Meteor. 25: 87-97.

Brine, D., Iqbal, M. (1983). Diffuse and global solar spectral irradiance under cloudless skies. Solar Energy, 30: 447-453.

Dai, Q., Fang, X. (2014). A simple model to predict solar radiation under clear sky conditions. Advances in Space Research. 53: $1239-1245$.

Fröhlich, C., London, J. (1986). Revised instruction manual on radiation instruments and measurements. Ginebra, Suiza: WCRP Publications.

Gónima, L. (1993). Simple algorithm for the atmospheric correction of reflectance images. International Journal of Remote Sensing. 14: 1179, 1187.

Goody, R. M., Yung, Y. L. (1989). Atmospheric Radiation: theoretical basis. New York, USA: Oxford University Press.

Hess, M., Koepke, P. Schult, I. (1998). Optical properties of aerosols and clouds: the software package OPAC. American Meteorological Society. 79: 831-844.

Iqbal, M. (1983). An introduction to solar radiation. Toronto, Canada: Academic Press.

Irvine, W. (1963). The asymmetry of the scattering diagram of a spherical particle. Astronomical Institutes of The Netherland. 17: 176-184.

Janjai, S., Nunez, M., Prathumsit, L., Wattam, R., Sabooding, R. (2013). A semi-empirical approach for the estimation of global, direct and diffuse illuminance under clear sky condition in the tropics. Energy and Buildings. 66: 177-182.

Justus, C., Paris, M. (1985). A model for solar spectral irradiance and radiance at the bottom and top of a cloudless atmosphere. Journal of Climate and Applied Meteorology. 24: 193-205.

Kasten, F. (1965). A new table and approximation formula for the relative optical air mass. Theoretical and Applied Climatology. 14: 206-223.

Leckner, B. (1978). The spectral distribution of solar radiation at the Earth's surface elements of a model. Solar Energy. 20: 143-150.
Lopez, G., Batlles, F., Tovar, J. (2007). A new simple parameterization of daily clear-sky global solar radiation including horizon effects. Energy Conversion and Management. 48: 226-233.

National Aeoronautics and Space Administration (NASA) (2015). Ozone \& Air Quality. Disponible en: ftp://toms. gsfc.nasa.gov/pub/omi/data/ozone/ accedida en noviembre de 2015.

Optical Properties of Aerosols and Clouds (OPAC). 2016. Fecha de consulta: Entre junio y diciembre de 2016. Disponible en: http://andromeda.caf.dlr.de/data-products/spectroscopydata/optical-properties-aerosols-and-clouds-opac.

Pacheco, A., Carlvalho, A., Aki, A., Morgan, E., Terra, F., Evaldo, A., Almeida, A. (2017). Global radiation by simplified models for the state of Mato Grosso, Brazil. Pesquisa Agropecuaria Brasileira. 52: 215-227.

Pelkowski, J. (2009). A physical rationale for generalized Ångström-Prescott regression. Solar Energy. 83: 955-963.

Prescott, J. (1940). Evaporation from water surface in relation to solar radiation. Transactions of the Royal Society of South Australia. 64: 114-118.

Russell, P., Bergstrom, R., Shinozuka, Y. Clarke, A., Decarlo, P., Jimenez, J., Livingston, J., Redemann, J., Dubovik, O., Strawa, A. (2010). Absorption Ångström Exponent in AERONET and related data as an indicator of aerosol composition. Atmospheric Chemistry and Physics. 10: 1155-1169.

Servicio Meteorológico Alemán. (2016). Disponible en ftp://ftpcdc.dwd.de/pub/CDC/, accedida entre enero de y junio de 2016.

Tang, W., Yang, K. He, J. Qin, J. (2010). Quality control and estimation of global solar radiation in China. Solar Energy. 84: 466-475.

Yang, K., Huang, G., Tamai, N. (2001). A hybrid model for estimating global solar radiation. Solar Energy. 70: 13-22.

Yang. K., Koike, T., Ye, B. (2006). Improving estimation of hourly, daily, and monthly solar radiation by importing global data sets. Agricultural and Forest Meteorology. 137: 43-55.

World Climate Programe (WCP). (1986). A preliminary cloudless standard atmosphere. WCP-No. 112. 